EPJ Web of Conferences 53, 01005 (2013)

DOI: $10.1051 /$ epjconf/20135301005

(C) Owned by the authors, published by EDP Sciences, 2013

\title{
The energy spectrum of cosmic rays at the highest energies
}

\author{
Bruce R. Dawson ${ }^{1}$, Ioana C. Mariş ${ }^{2}$, Markus Roth ${ }^{3}$, Francesco Salamida ${ }^{4}$, \\ Tareq Abu-Zayyad ${ }^{5}$, Daisuke Ikeda ${ }^{6}$, Dmitri Ivanov ${ }^{5,7}$, Yoshiki Tsunesada ${ }^{8}$, \\ Mikhail I. Pravdin ${ }^{9}$, Artem V. Sabourov ${ }^{9}$ for the Pierre Auger, \\ Telescope Array and Yakutsk Collaborations ${ }^{\mathrm{a}}$
}

${ }^{1}$ School of Chemistry \& Physics, The University of Adelaide, Adelaide, S.A., Australia

${ }^{2}$ Laboratoire de Physique Nucléaire et de Hautes Energies (LPNHE), Universités Paris 6 et Paris 7, CNRS-IN2P3, Paris, France

${ }^{3}$ Karlsruhe Institute of Technology - Campus North - Institut für Kernphysik, Karlsruhe, Germany

${ }^{4}$ Institut de Physique Nucléaire d'Orsay (IPNO), Université Paris 11, CNRS-IN2P3, Paris, France

${ }^{5}$ University of Utah, Department of Physics and High Energy Astrophysics Institute, Salt Lake City, Utah, USA

${ }^{6}$ Institute for Cosmic Ray Research, University of Tokyo, Kashiwa, Japan

${ }^{7}$ Rutgers-The State University of New Jersey, Department of Physics and Astronomy, Piscataway, New Jersey, USA

${ }^{8}$ Graduate School and Engineering, Tokyo Institute of Technology, Tokyo, Japan, Yu.G.

Shafer Institute of Cosmophysical Research and Aeronomy, Yakutsk, Russia

\begin{abstract}
One of several working groups established for this workshop was charged with examining results and methods associated with the UHECR energy spectrum. We summarize the results of our discussions, which include a better understanding of the analysis choices made by groups and their motivation. We find that the energy spectra determined by the larger experiments are consistent in normalization and shape after energy scaling factors are applied. Those scaling factors are within systematic uncertainties in the energy scale, and we discuss future work aimed at reducing these systematics.
\end{abstract}

\section{INTRODUCTION}

The energy spectrum working group (WG) was established approximately two months in advance of this workshop with membership from the Pierre Auger Observatory, the Telescope Array (TA) and the Yakutsk experiment. In addition, some of our members had been part of the HiRes collaboration, and there were strong links with the AGASA experiment. Our charge was to assess the current status of our knowledge of the UHECR energy spectrum, and to understand more clearly the analysis methods employed by the experiments, and their motivations.

All of the currently operational experiments (Auger, TA, Yakutsk) recognize the advantages of using an optical technique for calibrating the energy parameter of their surface detector arrays. Auger and TA use fluorescence light, while Yakutsk uses Cherenkov light. Apart from this common base, there are some significant differences in analysis methods.

aFor the full authorlist see Appendix "Collaborations" in this volume

This is an Open Access article distributed under the terms of the Creative Commons Attribution License 2.0, which permits unrestricted use, distribution, and reproduction in any medium, provided the original work is properly cited. 


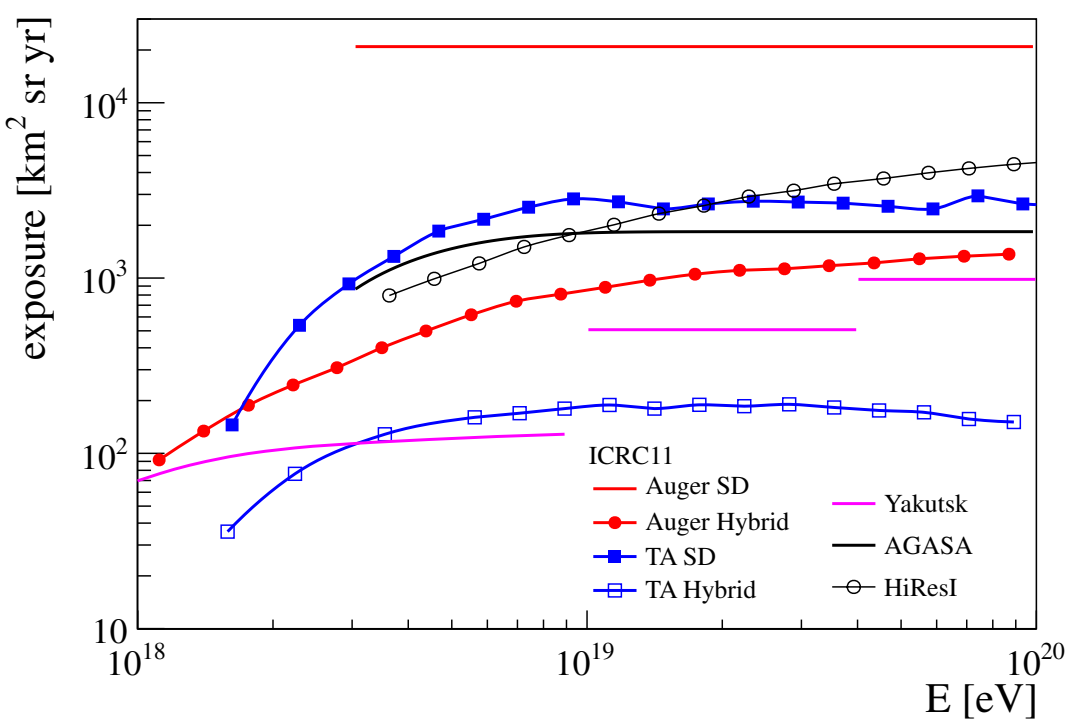

Figure 1. Exposures of the experiments at the time of the ICRC 2011 conference for Auger SD [1], Auger Hybrid [1], TA SD [2], TA Hybrid [3], Yakutsk SD [4], AGASA [5] and HiRes I [6]. After M. Unger [7].

We were pleased to confirm that there are no major inconsistencies between the energy spectra obtained by Auger, HiRes and TA. Future work will endeavor to understand the differences that do exist, and to take advantage of worldwide experience to hone our methods.

\section{SCOPE OF THE WORKING GROUP DISCUSSIONS}

At the beginning of the WG a framework was determined for its work.

1. Investigate the level of agreement in the normalization and shape of the UHECR energy spectrum determined by the groups;

2. Understand the methods for determining aperture and exposure, and compare the current exposures;

3. Understand the methods and motivations for determining energy via the surface detector (SD) of the experiments, including how zenith angle dependence (attenuation) is handled;

4. Discuss methods for fluorescence detector (FD)/optical calibration of the SD energy scale, and examine how these calibrations differ from those determined via simulation;

5. Examine the systematic uncertainties in the energy scale of each experiment, and discuss how these may be reduced in the future. If some systematics are common to the experiments, can we agree on using a particular method or measurement? One example of this is the fluorescence yield description.

During the two month working period, the WG communicated via a Wiki page, facilitating a collection of documents. The WG also held four productive Skype sessions which allowed an easy exchange of information.

\section{COMPARING ENERGY SPECTRUM MEASUREMENTS}

The exposures (in $\mathrm{km}^{2} \mathrm{sr} \mathrm{yr}$ ) of some past and current experiments are shown in Figure 1 as a function of energy. In the case of fluorescence detector spectra (Auger Hybrid, TA Hybrid and HiRes I), the exposure must be calculated using detector simulations and knowledge of the detector live time and 


\section{UHECR 2012}

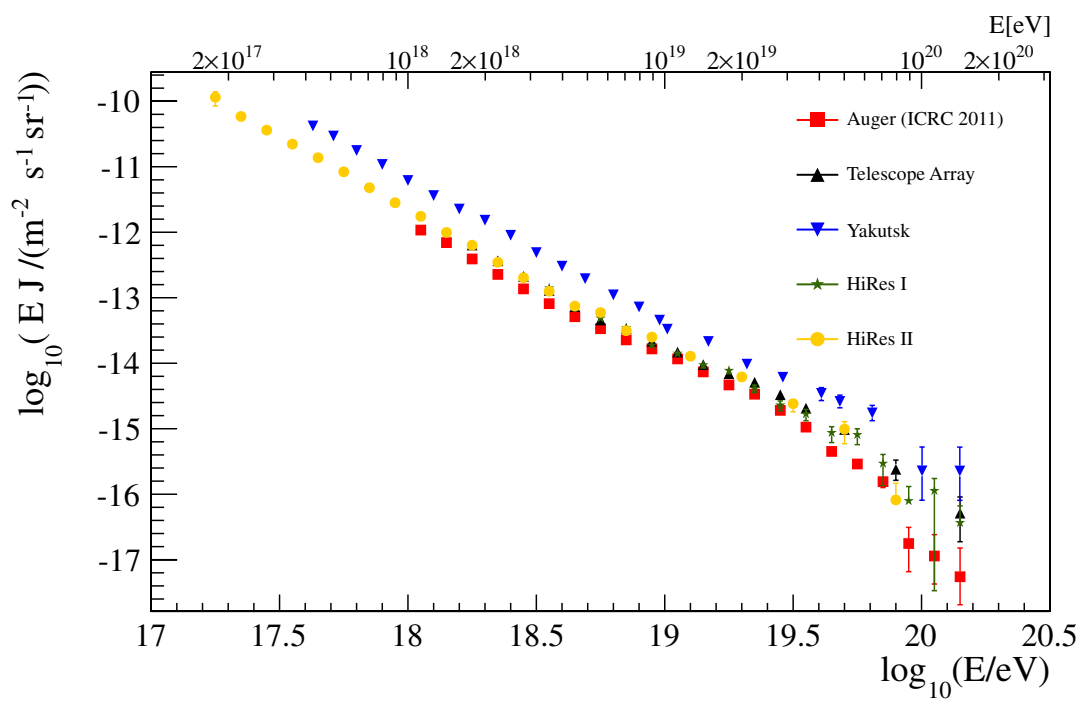

Figure 2. Published energy spectra, with the flux multiplied by $E$, for Auger (combined Hybrid/SD) [1], TA SD [2], Yakutsk SD [4], HiRes I [8], and HiRes II [8].

Table 1. Results of a triple power-law fit to the UHECR spectrum. Fit results are from AGASA [9], the combined HiRes I \& II mono spectra [8], Auger (Hybrid + SD) [1] and Telescope Array (SD) [2]. The fit to the Yakutsk SD spectrum [4] was done by the WG.

\begin{tabular}{llllll}
\hline & $\gamma_{1}$ & $\gamma_{2}$ & $\gamma_{3}$ & $\log _{10} E_{A}$ & $\log _{10} E_{S}$ \\
\hline AGASA & $3.16 \pm 0.08$ & $2.78 \pm 0.3$ & - & 19.01 & \\
Yakutsk & $3.29 \pm 0.17$ & $2.74 \pm 0.20$ & - & $19.01 \pm 0.01$ & - \\
HiRes & $3.25 \pm 0.01$ & $2.81 \pm 0.03$ & $5.1 \pm 0.7$ & $18.65 \pm 0.05$ & $19.75 \pm 0.04$ \\
Auger & $3.27 \pm 0.02$ & $2.68 \pm 0.01$ & $4.2 \pm 0.1$ & $18.61 \pm 0.01$ & $19.41 \pm 0.02$ \\
TA & $3.33 \pm 0.04$ & $2.68 \pm 0.04$ & $4.2 \pm 0.7$ & $18.69 \pm 0.03$ & $19.68 \pm 0.09$ \\
\hline
\end{tabular}

atmospheric conditions. Such exposures generally increase with energy, though in the case of TA Hybrid the exposure saturates because it is limited by the aperture of the surface detector array. Surface detector exposures are generally more robust, because they are calculated by integrating the geometrical aperture of the surface array over the live time of the experiment.

The published energy spectra are shown in Figure 2. This particular representation, with the flux axis multiplied by a single power of energy, is related to the actual measurement where a number of air showers is detected in a particular logarithmic energy interval, $\left(\frac{d N}{d \log _{10} E}=\frac{d N}{d E} E \ln 10 \propto J E\right)$. Systematic differences are apparent. However all experiments shown here detect the spectral flattening at the ankle. With the exception of Yakutsk (due to lower exposure), all the experiments observe a suppression, perhaps the GZK cut-off, at the highest energies.

A traditional way to characterize spectra is via a broken power-law fit with two break-points at the ankle energy $E_{A}$ and the beginning of the suppression at energy $E_{S}$. Around these energies the spectrum has a power law form $E^{-\gamma}$ with indices $\gamma_{1}$ below the ankle, $\gamma_{2}$ between the ankle and the suppression, and $\gamma_{3}$ above the suppression. The parameters are shown in Table 1. The fits were done by the various collaborations (references listed in the caption) except for Yakutsk, where the fit was done by the WG. The HiRes fits were done after combining the mono spectra from HiRes I \& II [8].

We have included results from AGASA in this table, given its importance in our field. As is well known, the AGASA energy spectrum [9] did not show evidence for a high-energy suppression, 


\section{EPJ Web of Conferences}
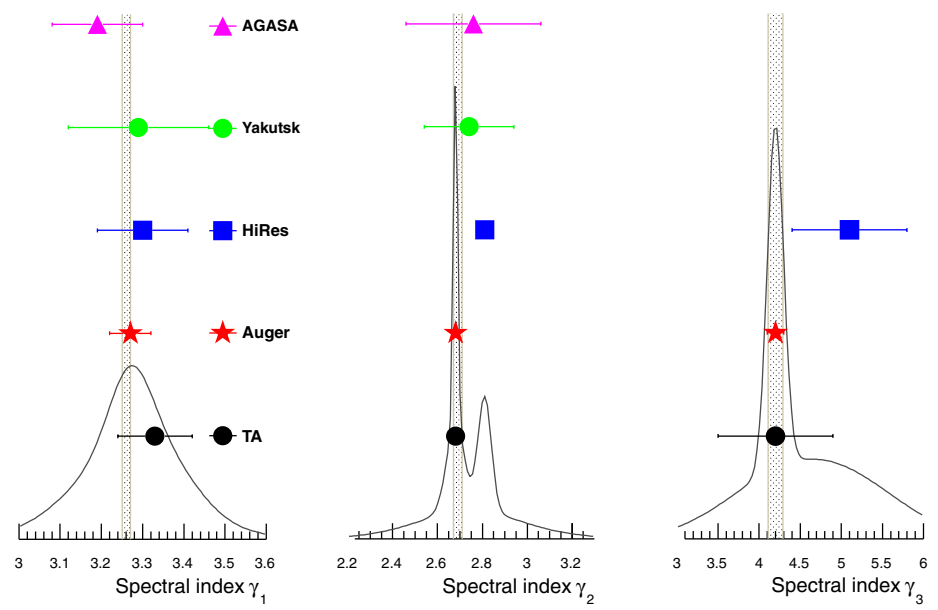

Figure 3. Spectral indices for the triple-power law fits from Table 1. The solid black line is the sum of the Gaussian probability distributions implied by the errors in the indices. The shaded bar represents the weighted mean of the measurements and its uncertainty.
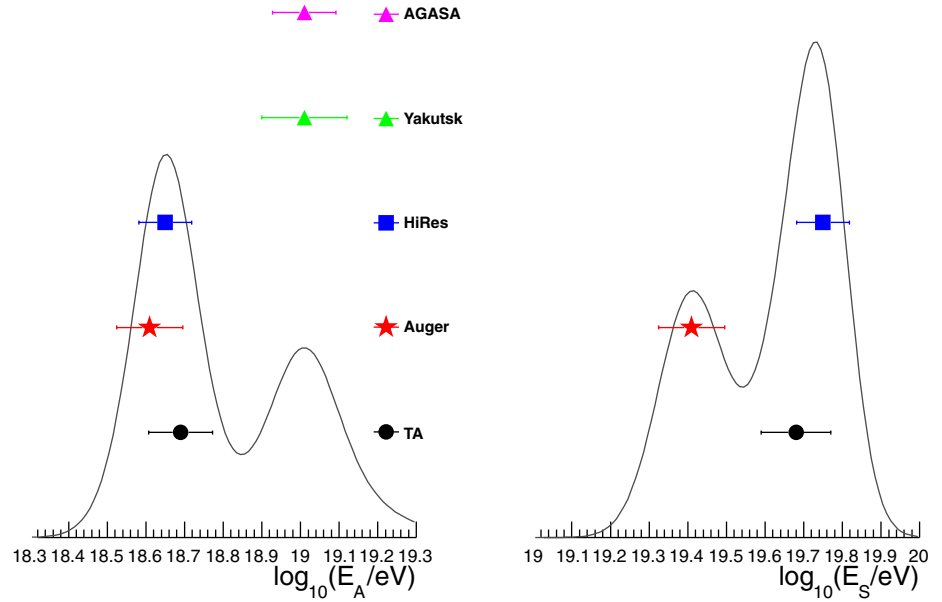

Figure 4. Break-point energies for the triple-power law fits from Table 1. The solid black line is the sum of the Gaussian probability distributions implied by the errors in the break-point energies. Those errors are the statistical uncertainty folded with the systematic uncertainty in the energy scale.

in contrast with HiRes and Auger. This was part of the motivation behind the formation of the Telescope Array experiment, a combination of fluorescence detectors and a plastic scintillator surface detector.

These fits results are displayed graphically in Figure 3 and Figure 4. We use the quoted uncertainties of the fits to produce a probability distribution for each parameter. We observe that there is general agreement on the values of spectral indices, with the possible exception of $\gamma_{2}$. There is more disagreement on the positions of the break-points in Figure 4.

The energies relating to spectral features can be affected by experimental energy resolution and by systematic energy uncertainties. We discuss each of these in turn. 


\section{UHECR 2012}

\subsection{Treatment of detector resolution}

The differential cosmic ray intensity in a given energy interval is determined experimentally by $n_{i}=N_{\mathrm{obs}}^{i} / A_{0} T$, where $A_{0}$ is the geometrical aperture of the detector (area $\times$ solid angle), $T$ is the observation time (the exposure is defined as $\mathcal{E}=A_{0} T$ ), and $N_{\mathrm{obs}}^{i}$ is the number of observed cosmic ray events in the energy bin $i$ in the duration $T$. Because of the energy resolution determined by the detector performance and the analysis algorithm, however, bin-to-bin migrations affect the number of events in the energy bins and hence the energy spectrum.

The effect of energy resolution on the spectrum is treated slightly differently by the existing experiments. As was the practice with HiRes, the Telescope Array experiment corrects for the resolution by including its effects into the exposure calculation, for both the surface detector spectrum and their hybrid spectrum. They begin by generating Monte Carlo showers (QGSJET-II model) assuming a pure proton composition and the spectrum determined by HiRes [8], then passing the showers through a detailed detector simulation. Comparisons of various real data distributions with those from simulations give confidence in the simulations. The exposure for each energy bin is then calculated as $\left(N_{\text {rec }} / N_{\text {th }}\right) A_{0} T$ where $N_{\text {rec }}$ is the number of reconstructed events (using the reconstructed energy), and $N_{\text {th }}$ is the number of thrown events (using the generated energy). Since the resulting TA SD spectrum is very consistent with the HiRes spectrum assumed in the process, the bias due to this choice of spectrum in the unfolding is negligible. The assumption of a pure proton composition is based on the results of the HiRes experiment, and the systematic uncertainty connected with this choice is small for $\log _{10}(E / \mathrm{eV})>18.5$.

For the Auger spectra, the resolution effects are corrected using a forward-folding method. The true flux description is based on a fit to the measured Auger spectrum, assuming a combination of power laws and a Fermi function-like suppression (e.g. Figure 5 of [1]). The migration matrix is built from CORSIKA (QGSJet II.3/Fluka) with GEANT4 detector simulations, assuming a 50:50 mixed composition of proton and iron sample showers. The energy of the simulations is scaled to take into account the known energy-scale discrepancy with real data [13]. Distributions of shower observables (e.g. zenith angle, stations per event, signal per station, $\chi^{2}$ of LDF fit, energy resolution) are well matched by MC results. The correction contributes a systematic uncertainty to the flux of $5 \%$, calculated assuming no energy re-scaling, and pure compositions. The systematic uncertainty due to the choice of the true spectrum in the simulation is negligible.

In the case of Yakutsk, no correction has yet been made to the spectrum for the effects of resolution.

\subsection{Budget of the systematic energy uncertainty}

The energy scale for the Yakutsk SD spectrum is calibrated by atmospheric Cherenkov light observations on a subset of showers. As a reminder, we point out that the Yakutsk SD energy parameter is either $S_{300}$ for the trigger-500 array (the particle density $300 \mathrm{~m}$ from the core) or $S_{600}$ for the trigger-1000 array. An attenuation correction, derived from a constant intensity cut (CIC) method (and cross-checked using Cherenkov measurements) is applied to convert $S_{300}$ or $S_{600}$ to their equivalents for a vertical shower. Finally, the primary particle energy is determined from

$$
\begin{aligned}
& E_{0}=(6.5 \pm 1.6) \times 10^{16} S_{300}\left(0^{\circ}\right)^{0.940 .02} \mathrm{eV} \\
& E_{0}=(4.6 \pm 1.2) \times 10^{17} S_{600}\left(0^{\circ}\right)^{0.980 .02} \mathrm{eV}
\end{aligned}
$$

where the constants are derived from the relationship between the $S_{300}\left(0^{\circ}\right)$ or $S_{600}\left(0^{\circ}\right)$ and $Q_{400}$, the Cherenkov light intensity at $400 \mathrm{~m}$ from the core [4]. The systematic error in energy implied by these conversions is approximately $25 \%$, attributed to calibration systematics in the Cherenkov detectors and systematics in light transmission through the atmosphere [4]. A study has been done replacing the Cherenkov calibration by one that converts $S_{300}$ or $S_{600}$ to energy via simulations only [10]. This results in $E_{0}=(2.7-3.0) \times 10^{17}\left(S_{600}\right)^{0.99} \mathrm{eV}$, giving energies that are only about $60 \%$ of those derived using the Cherenkov light measurements. Interestingly, this disagreement between shower simulations 


\section{EPJ Web of Conferences}

Table 2. Estimates of contributions to systematic uncertainties in the fluorescence energy scale, for HiRes [8], Auger [11] and the Telescope Array [3]. The total is the sum of the uncertainties in quadrature.

\begin{tabular}{lccc}
\hline & HiRes & Auger & TA \\
\hline Photometric calibration & $10 \%$ & $9.5 \%$ & $10 \%$ \\
Fluorescence Yield & $6 \%$ & $14 \%$ & $11 \%$ \\
Atmosphere & $5 \%$ & $8 \%$ & $11 \%$ \\
Reconstruction & $15 \%$ & $10 \%$ & $10 \%$ \\
Invisible Energy & $5 \%$ & $4 \%$ & incl. above \\
\hline TOTAL & $\mathbf{1 7 \%}$ & $\mathbf{2 2 \%}$ & $\mathbf{2 1 \%}$ \\
\hline
\end{tabular}

and a calorimetric experimental method (Cherenkov light in this case) is in the opposite direction to those found in the other experiments (see below). In any case, for the Yakutsk data discussed in the present paper, we assume the original, Cherenkov light-based calibration from the original spectrum publication [4].

In the Auger SD analysis, the ground parameter used to extract the primary energy is $S(1000)$, the water Cherenkov tank signal at $1000 \mathrm{~m}$ from the core. Through an attenuation correction derived from the CIC method, $S(1000)$ is converted to $S_{38}$, the value of $S(1000)$ the shower would have if recorded at the median zenith angle of $38^{\circ}$ [11]. This parameter is then related to the primary energy using fluorescence observations of a subset of showers, taking advantage of a near-calorimetric fluorescence energy determination. In these ways, the energy assignment is nearly free of simulations, with the exception being in the estimation of a small (of order 10\%, see below) correction for invisible energy, that part of the primary energy carried into the ground by neutrinos and high-energy muons that does not result in full fluorescence emission.

The Telescope Array SD analysis methods are broadly similar to that of AGASA [5], with the ground array energy parameter being $S(800)$, the scintillator signal at $800 \mathrm{~m}$ from the core. TA uses simulations to determine the change in $S(800)$ as a function of shower zenith angle at fixed energy. The first energy estimate $E_{\mathrm{SD}}$ from $S(800)$ is rescaled by using the average FD-SD energy scale ratio obtained from hybrid events, as $E=\left\langle E_{\mathrm{FD}} / E_{\mathrm{SD}}\right\rangle_{\mathrm{h}} E_{\mathrm{SD}}$, where $\left\langle E_{\mathrm{FD}} / E_{\mathrm{SD}}\right\rangle_{\mathrm{h}}=1 / 1.27$ [2, 12]. The use of MC simulations is to account for any changes in the attenuation function with energy, given that the CIC method is best applied at lower energy where the statistical uncertainties are smaller. On the other hand, the simulation route is subject to uncertainties in both the choice of hadronic model and the mass composition assumption. (The Auger collaboration has applied the CIC method with increasing cuts on energy in an attempt to see any changes in the assumed attenuation with zenith angle, but so far no significant change has been detected.)

Auger and the Telescope Array both take great care in determining the energy scale of fluorescence measurements, as this is the basis of the energy measurements for both hybrid and SD spectra. While the fluorescence technique is conceptually elegant, with the amount of light produced being directly proportional to the energy deposited by the shower in the atmosphere, there are practical challenges. Some of these are expressed through estimates of the systematic uncertainties related to the energy scale, listed in Table 2 for the two experiments and for HiRes. Photometric calibration refers to the absolute calibration of the telescopes and photomultipliers, and their wavelength response; uncertainties in the fluorescence yield include those on the absolute efficiency, its wavelength dependence, and its dependence on pressure, temperature and humidity; atmospheric uncertainties include those relating to Rayleigh and aerosol scattering; reconstruction uncertainties are mainly related to the efficiency of light collection in the telescope cameras; and the invisible energy uncertainties are based on lack of knowledge of the true mass composition and on the spread of predictions of invisible energy by different hadronic models. The total systematic uncertainty on the fluorescence energy is of order $20 \%$ for the three experiments.

We will return to aspects of the fluorescence energy scale after examining the level of agreement between the published energy spectra. 


\section{UHECR 2012}

\section{COMPARING ENERGY SCALES}

The WG undertook an exercise to see if the various spectra could be brought into better agreement through a simple scaling of the energy scale. This assumes that any current disagreement is based solely on the energy scale, and not on other factors such as aperture calculation or the treatment of energy resolution, but we believe that the results are informative. As input to the calculation we took energy spectra published by the Yakutsk, HiRes, Telescope Array and Auger collaborations. Then, to obtain the energy normalizations for the individual energy spectra, we performed a minimization of the following $\chi^{2}$ function to compare one spectrum (called the reference spectrum) to one from another experiment,

$$
\chi^{2}=\sum_{i=0}^{i<N_{0}} \frac{\left(F_{0, i}-\hat{F}_{k}\left(x_{0, i}^{0}-\log _{10} \alpha_{k}\right)\right)^{2}}{\sigma_{0, i}^{2}+\hat{\sigma}^{2}\left(x_{0, i}-\log _{10} \alpha_{k}\right)}+\frac{\left(1 .-\alpha_{k}\right)^{2}}{\sigma_{s y s, k}^{2}+\sigma_{s y s, 0}^{2}}
$$

where the parameters are defined below,

- $k$ is the index of the experiment, $k \in\{0,1,2,3\}$, where $k=0$ represents the energy spectrum of the reference experiment

- $N_{k}$ is the number of data points for the $k$-th spectrum, and $i$ is the index for the data points, $i \in\left\{0, \ldots, N_{k}-1\right\}$

- $E_{k, i}$ is the energy and $J_{k, i}$ is the differential flux of the $i$-th data point

- $\left(F_{k, i}, x_{k, i}\right)$ are the data points for the fit, with $x_{k, i}=\log _{10}\left(E_{k, i}\right)$ and $F_{k, i}=E_{k, i} J_{k, i}$

- $\sigma_{k, i}$ is the statistical uncertainty of $F_{k, i}$

- $\alpha_{k \in\{0, \ldots, 3\}}$ are the multiplicative scaling factors for the energy. We fit for $\log _{10}\left(\alpha_{k \in(0,3)}\right)$ since this factor is symmetric when comparing the shifts of two spectra against each other.

- $\hat{F}, \hat{\sigma}$ is the flux multiplied by energy, and its uncertainty, estimated for $k>0$ from a linear interpolation in log-log space between neighboring bins

- $\sigma_{s y s, k}$ represent the energy systematic uncertainties, taken as $22 \%$ for the Telescope Array and Pierre Auger measurements, 25\% for the Yakutsk measurement and 17\% for the HiRes experiment.

For differential spectra $J(E)$, we compared $F=E J(E)$ from the different experiments, because $E J(E) \propto E d N / d E \propto d N / d\left(\log _{10} E\right)$ is what is actually measured by the experiments. Because there is an assumption in this $\chi^{2}$ minimization that the uncertainties on the variables are normally distributed, we perform the fit only up to $\log _{10}(E / \mathrm{eV})=19.5$ to avoid the Poisson statistics of low event counts. The spectra investigated are listed in Table 3 with their publication references, together with the results of this scaling exercise. Each column is for a particular reference spectrum, and the fitting was done in each case for just the two spectra indicated by the column and the row. For each fit, the numbers represent $\log _{10} \alpha$ and its uncertainty. The reference spectra were taken to be those of the currently operating experiments, and the final column uses an average of the Auger and TA spectra as the reference, a convenient baseline defined by the WG. The rescaled energy spectra, referenced to the average of TA and Auger, are illustrated in Figures 5 \& 6.

Examining Figures $5 \& 6$ and the $\chi^{2}$ values in the body of Table 3, we see that the rescaled spectra are in very good agreement in both shape and normalization. We searched for evidence of energy dependent scaling factors of the form $\alpha(E / \mathrm{EeV})^{\beta}$ and found values of $\beta$ consistent with zero. With the energy scaling, it is not surprising that the positions of the spectral features are now more consistent, as shown in Figure 7.

Overall we find that the energy scales of the TA and HiRes experiments are nearly identical, and that TA and Auger have a an energy scale difference expressed as $\log _{10} \alpha= \pm 0.085$, or that energies are reconstructed by Auger at values $\sim 20 \%$ smaller than TA for the same flux. Such a difference is entirely consistent with the systematic energy uncertainties listed in Table 2, a topic we will return to in the next section. We also find that Yakutsk energies are overestimated by $60 \%$ with respect to TA and $95 \%$ with respect to the Auger scale. These inconsistencies are more difficult to explain in terms of estimated systematic uncertainties, which are estimated to be $25 \%$ for the Yakutsk Cherenkov light 


\section{EPJ Web of Conferences}

Table 3. Parameters obtained from the energy-shift fitting for pairs of spectra. Publication references for the spectra are given in the left-hand column. We fitted for the logarithm of the scaling factors, with the entries representing $\log _{10}(\alpha)$ and its uncertainty. The value of the $\chi^{2}$ /ndof and its probability are also given. The columns indicate the reference spectrum, chosen from the currently operating experiments, including in the last column an average of the Auger and TA spectra (a convenient reference in the opinion of the WG). The differences in the numbers for a given pair of spectra when switching the reference spectrum is due to the interpolations used in the method.

\begin{tabular}{|c|c|c|c|c|}
\hline \multicolumn{4}{|c|}{ Reference experiment } & \multirow{2}{*}{$\begin{array}{r}\text { Averaged reference } \\
\langle\text { Auger, TA }\rangle\end{array}$} \\
\hline & TA & Auger & Yakutsk & \\
\hline TA & - & $0.0851 \pm 0.0027$ & $-0.2087 \pm 0.0056$ & $0.0423 \pm 0.0027$ \\
\hline \multirow[t]{2}{*}[2]{} & - & $\chi^{2} /$ ndof $=0.5060$ & $\chi^{2} /$ ndof $=0.5078$ & \\
\hline & - & $P=0.9874$ & $P=0.9751$ & \\
\hline \multirow{3}{*}{$\begin{array}{c}\text { Auger } \\
{[1]}\end{array}$} & $-0.0843 \pm 0.0028$ & - & $-0.2951 \pm 0.0048$ & $-0.0423 \pm 0.0027$ \\
\hline & $\chi^{2} / \mathrm{ndof}=0.5671$ & - & $\chi^{2} /$ ndof $=0.7478$ & \\
\hline & $P=0.9586$ & - & $\mathrm{P}=0.8227$ & \\
\hline \multirow{3}{*}{$\begin{array}{l}\text { Yakutsk } \\
\text { [4] }\end{array}$} & $0.2110 \pm 0.0043$ & $0.2985 \pm 0.0038$ & - & $0.2562 \pm 0.0038$ \\
\hline & $\chi^{2} /$ ndof $=0.6590$ & $\chi^{2} /$ ndof $=0.9550$ & - & \\
\hline & $P=0.9000$ & $P=0.5342$ & - & \\
\hline \multirow{3}{*}{$\begin{array}{l}\text { HiRes I } \\
\text { [8] }\end{array}$} & $-0.0009 \pm 0.0063$ & $0.0829 \pm 0.0047$ & $-0.1978 \pm 0.0103$ & $0.0406 \pm 0.0047$ \\
\hline & $\chi^{2} /$ ndof $=0.4604$ & $\chi^{2} /$ ndof $=0.6530$ & $\chi^{2} /$ ndof $=0.5009$ & \\
\hline & $\mathrm{P}=0.9901$ & $\mathrm{P}=0.9144$ & $\mathrm{P}=0.9855$ & \\
\hline \multirow{3}{*}{$\begin{array}{l}\text { HiRes II } \\
\text { [8] }\end{array}$} & $-0.0003 \pm 0.0053$ & $0.0865 \pm 0.0049$ & $-0.2093 \pm 0.0033$ & $0.0442 \pm 0.0049$ \\
\hline & $\chi^{2} / \mathrm{ndof}=0.5135$ & $\chi^{2} /$ ndof $=0.3497$ & $\chi^{2} /$ ndof $=1.1036$ & \\
\hline & $\mathrm{P}=0.9784$ & $P=0.9993$ & $P=0.3233$ & \\
\hline
\end{tabular}

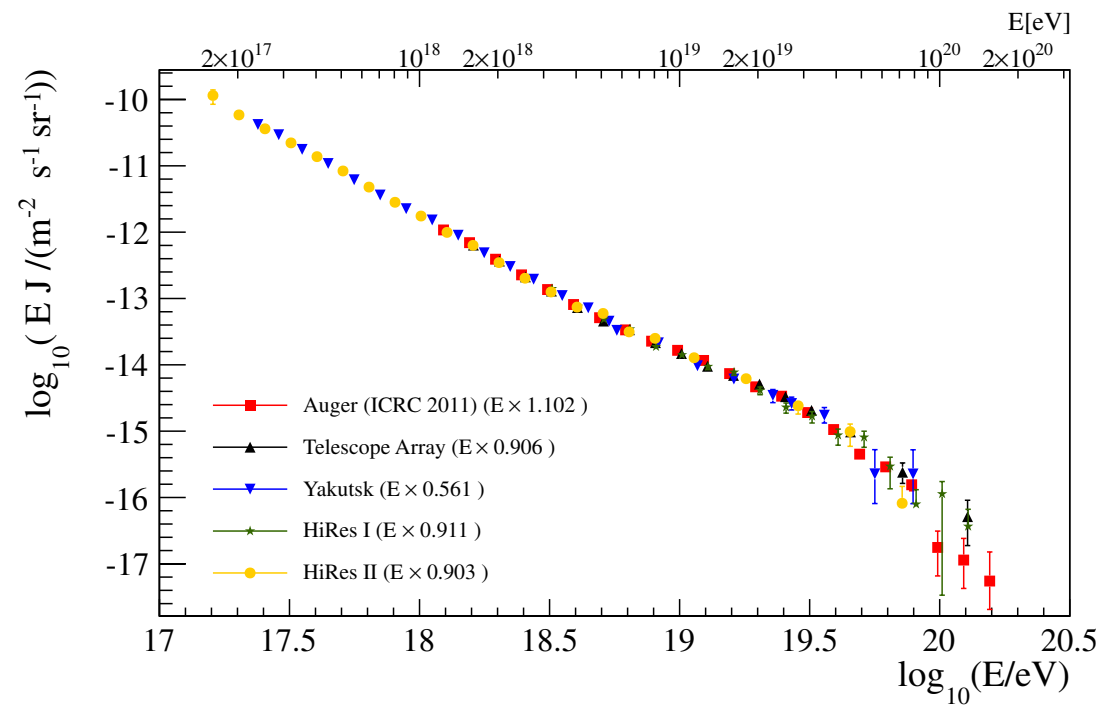

Figure 5. Spectra from Figure 2 after energy-rescaling. The reference spectrum is the average of those from Auger and TA. The values of $\log _{10} \alpha$ are given in Table 3, and the values of $\alpha$ are indicated on the plot.

calibration. We note that the alternative Yakutsk calibration procedure based on shower simulations described earlier (Section 3.2) would be more consistent with the fluorescence energy determinations.

This statement should not be taken to suggest that air shower simulations are superior to calibrating with calorimetric methods such as Cherenkov or fluorescence light. There are large uncertainties on the hadronic interaction model, and in calculations done by the TA and Auger experiments, inconsistencies 
UHECR 2012

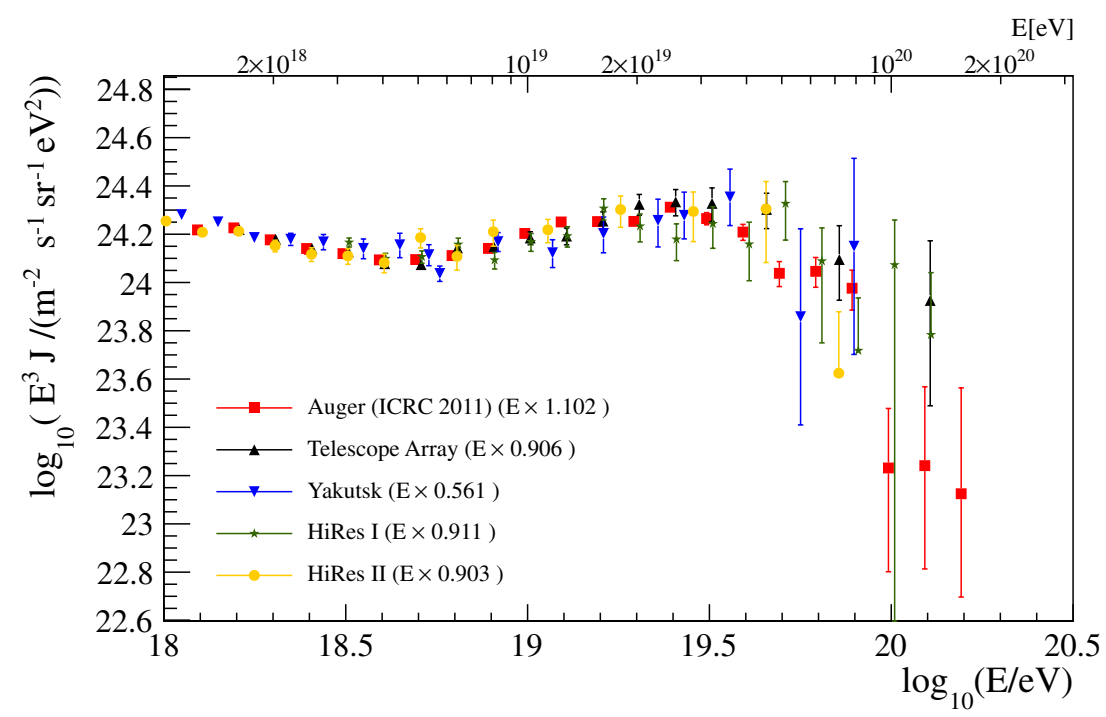

Figure 6. Re-scaled spectra from Figure 5, but in the form $E^{3} J$.
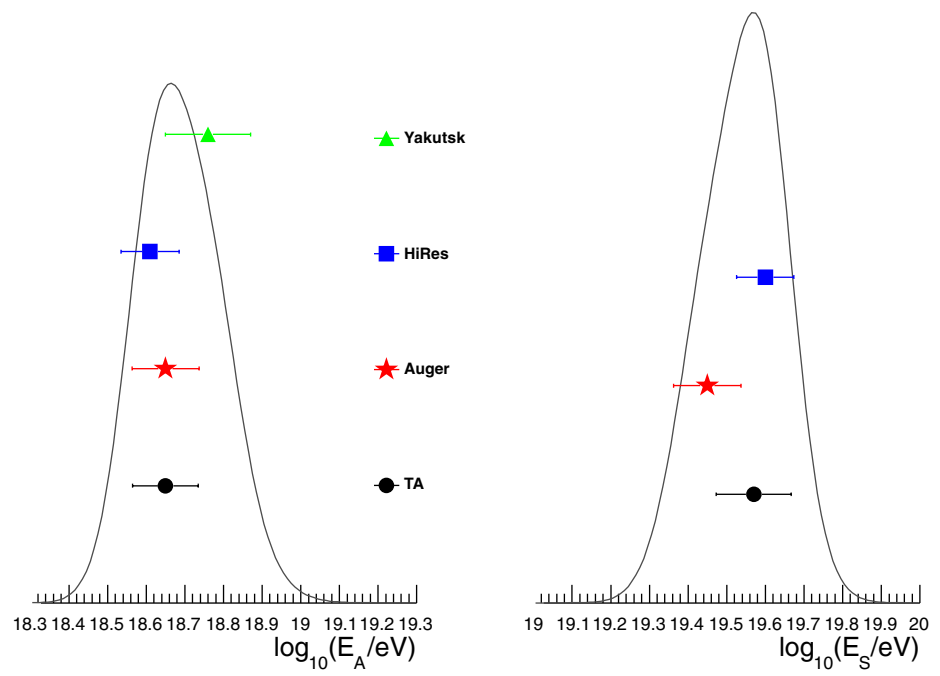

Figure 7. Break-point energies for the triple-power law fits after energy rescaling, to be compared with the original positions in Figure 4. The error bars again represent the statistical uncertainty folded with the systematic uncertainty in the energy scale for each experiment. The reference spectrum is the average of Auger and TA.

are also seen between a simulation energy scale and that derived from fluorescence measurements. The size of the discrepancy is dependent on the hadronic model and the mass composition assumptions. In TA, simulations of proton showers using QGSJET-II predict a energy for a given $S(800)$ which is $27 \%$ lower than the energy derived from fluorescence observations [2, 12]. Auger also sees an effect in the same direction, with simulations (protons or iron) underestimating the ground signal for a given (fluorescence-derived) energy [13]. Note that the simulation/fluorescence energy difference seen by Auger and TA is in the opposite direction to that seen in the simulation/Cherenkov comparison of Yakutsk. 

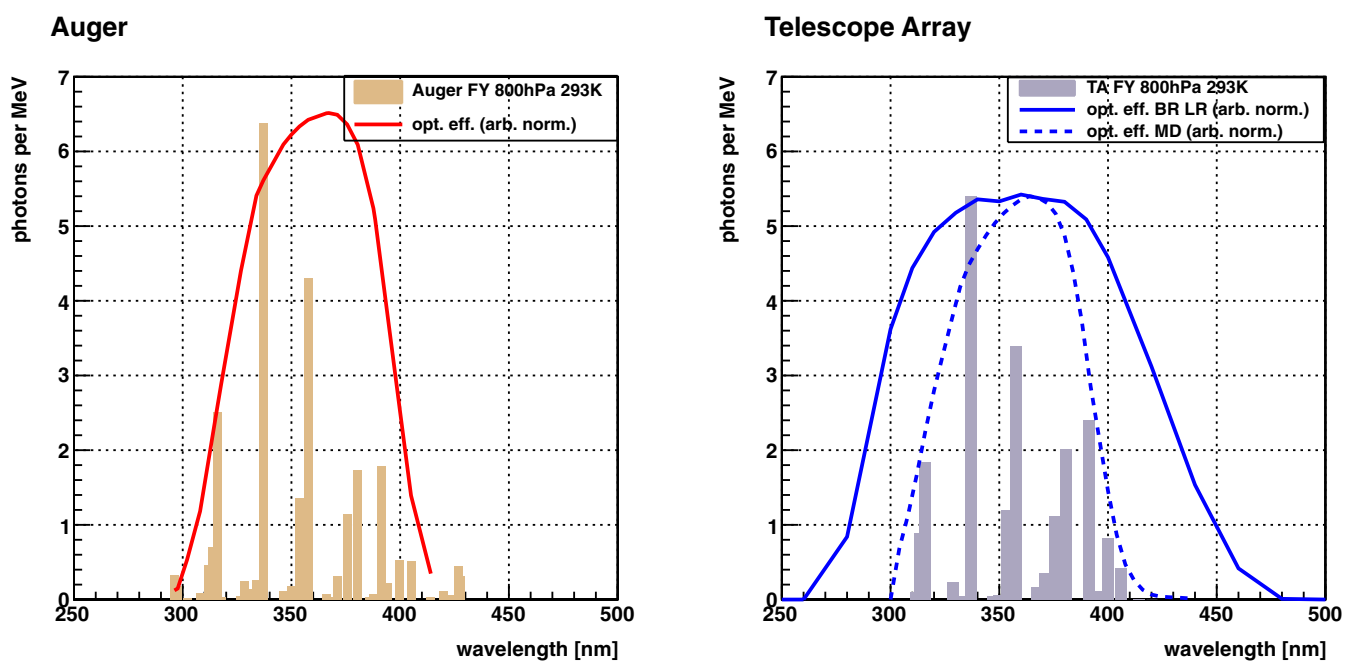

Figure 8. Optical efficiency curves (arbitrary normalization) together with the assumed fluorescence spectra for Auger and the Telescope Array. The spectra are for dry air at a pressure of $800 \mathrm{hPa}$ and a temperature of $293 \mathrm{~K}$. In the case of the Telescope Array, there are different optical efficiency curves for the Black Rock and Long Ridge sites on one hand, and the former HiRes telescopes now at the Middle Drum site on the other.

\subsection{Analysis \& calibration differences}

The comparisons of spectra in the previous section suggest that a simple rescaling of energy can bring the results into agreement. In the case of the Yakutsk/TA and Yakutsk/Auger comparisons the required rescaling is somewhat outside that allowed by the known systematic uncertainties, but Auger/TA rescaling is perfectly consistent with the $22 \%$ and $21 \%$ energy scale systematics of Auger and TA respectively (Table 2). In this section we concentrate on some differences in the analysis methods of Auger and TA relevant to the energy scale.

\subsubsection{Fluorescence yield}

The two experiments currently use different laboratory measurements of the fluorescence yield, its wavelength dependence, and its dependence on pressure, temperature and humidity. The Auger approach is outlined in [11]; it can be summarized as using the 2004 measurements of Nagano et al. for the yield of the $337 \mathrm{~nm}$ band, with the relative wavelength spectrum and its dependence on pressure, temperature and humidity coming from the AIRFLY collaboration. In contrast, TA uses spectral lines and their relative intensities from the FLASH experiment [14], but rescales these so that the total yield between $300-400 \mathrm{~nm}$ is consistent with [15], see [12]. Pressure and temperature corrections are also taken from [15], and no humidity correction is currently applied.

The telescopes of Auger and TA have a particular spectral response, and this must be taken into account when attempting to understand the effect of different fluorescence yield models. We refer to the spectral response of a telescope as its "optical efficiency", defined as the product of mirror reflectivity, optical filter transmission and PMT quantum efficiency as a function of wavelength. In the case of TA this also includes the "paraglas" window transmission, and in the case of Auger it includes the transmission of the corrector ring optics. Figure 8 shows the relative optical efficiency curves for Auger and TA, together with the assumed fluorescence spectra for the two experiments, for a pressure of $800 \mathrm{hPa}$ and a temperature of $293 \mathrm{~K}$. The TA experiment includes former HiRes telescopes at the Middle Drum site, so two optical efficiency curves are shown. The optical efficiency for the TA Long Ridge and 


\section{UHECR 2012}

Black Rock sites has a wider bandpass than either the Auger or the Middle Drum optical efficiencies. Such a bandpass increases the signal available, but also increases the level of night sky background. The two collaborations have decided to optimize their sensitivity in different ways.

Given the diversity in laboratory measurements of the fluorescence spectrum and normalization, it would be fair to conclude that we do not yet know the true fluorescence description, though progress is being made on this front [16]. However, we do have two possible versions of it in the models applied by Auger and TA. As an exercise, the WG considered the following question: "What would be the effect on one experiment's energy scale if the fluorescence model assumed by the other experiment was actually the "true" one?". To do this we first ignored the effect of atmospheric scattering in changing the shape of the fluorescence spectrum, and calculated the following "signal",

$$
S=\sum_{\lambda} Y(\lambda) \epsilon(\lambda)
$$

where $Y(\lambda)$ is the fluorescence spectrum and $\epsilon(\lambda)$ is the optical efficiency, both taken from Figure 8, with $\epsilon(\lambda)$ normalized to 1 at $375 \mathrm{~nm}$. As an example, the value of $S$ for the Auger spectrum and efficiency is 18.1 "signal" units. For a given experiment, we fixed the $\epsilon$ curve, but were able to switch the $Y$ curve.

First, we consider the possibility that the "true" fluorescence model is the TA description. Then for a shower of some energy $E_{0}$, Auger would record a signal of $S=\sum Y_{\mathrm{TA}} \epsilon_{A}=16.1$ units, while it would expect, on the basis of its assumed fluorescence model, to receive $S=\sum Y_{\mathrm{A}} \epsilon_{A}=18.1$ units. Here, the subscripts on $Y$ or $\epsilon$ refer to either "TA" or "A" for Auger. As a result, Auger will reconstruct an energy which is too low by $1-(16.1 / 18.1)=11 \%$ or $E_{\mathrm{TA}} / E_{\mathrm{A}}=1.12$.

Now consider that the "true" yield description is the Auger model. In this case, TA will receive from a shower of some energy a signal of $S=\sum Y_{\mathrm{A}} \epsilon_{\mathrm{TA}}=22.7$ units, while expecting a signal of $S=\sum Y_{\mathrm{TA}} \epsilon_{\mathrm{TA}}=19.4$ units. As a result TA will reconstruct an energy which is too high by $(22.7 / 19.4)-1=17 \%$, or $E_{\mathrm{TA}} / E_{\mathrm{A}}=1.17$. (Here we used the Black Rock and Long Ridge optical efficiency. When using the Middle Drum (HiRes) efficiency, we get a similar result of $E_{\mathrm{TA}} / E_{\mathrm{A}}=1.14$ ).

The actual numbers depend on which (if either) of the fluorescence models is the true one. But note that in both cases, the Auger reconstructed energy is lower than the TA reconstructed energy, in agreement with the direction of the discrepancy in the energy spectra. However, so far we have ignored the effect of the atmosphere in two ways; first the effect of atmospheric transmission, especially Rayleigh scattering, which will preferentially attenuate shorter wavelengths; and secondly the effect of humidity quenching of fluorescence, taken into account by Auger but not yet by TA. The first effect was tested by reconstructing a sample of real Auger air showers $\left(\log _{10}(E / \mathrm{eV})>18.5\right)$ using the TA fluorescence description, properly taking into account the differential attenuation across the fluorescence spectrum. The result derived above ignoring attenuation, $E_{\mathrm{TA}} / E_{\mathrm{A}}=1.12$ was reduced to $E_{\mathrm{TA}} / E_{\mathrm{A}}=1.08$ here. The effect of average levels of humidity is to reduce the yield by $5 \%$ and hence increase the reconstructed energies by 5\% [17]. Because Auger applies this correction and TA doesn't, the expected gap between $\mathrm{TA}$ and Auger energies is further reduced to $E_{\mathrm{TA}} / E_{\mathrm{A}}=1.03$. All these figures assume that the true fluorescence model is the TA one.

To summarize, if the true yield model is the TA one, TA and TA (Middle Drum) would reconstruct the correct energy, and Auger's energy would be low by about 3\%, ignoring all other effects. If the true yield model is the Auger one, Auger's energies would be correct, and TA energies would be too high by $8 \%$, and TA (Middle Drum) energies too high by 5\%. Similar results have been determined by Vazquez et al. [19].

\subsubsection{Atmospheric monitoring}

The atmosphere attenuates fluorescence and Cherenkov light primarily via Rayleigh and aerosol scattering, and via cloud cover. The density and temperature profile of the atmosphere determines the fluorescence yield, and the density profile governs the conversion of height to atmospheric depth. 


\section{EPJ Web of Conferences}

Table 4. Some highlights of the atmospheric monitoring activities at the former HiRes site, and at the Auger Observatory and the Telescope Array. The percentages in the left-hand column refer to the estimated systematic uncertainties in energy attributed to the atmosphere. References are also given in the left-hand column. CLF refers to "Central Laser Facility".

\begin{tabular}{|c|c|c|c|c|c|}
\hline & Radiosonde & LIDAR & CLF & IR Camera & Other \\
\hline $\begin{array}{l}\text { HiRes } \\
(5 \%) \\
{[20]}\end{array}$ & $\begin{array}{c}\text { Stereo: public data, } \\
\text { Salt Lake City \& } \\
\text { Elko, 200km from } \\
\text { site. Mono: US } \\
\text { standard atmos. }\end{array}$ & N/A & $\begin{array}{c}\text { Stereo:hourly } \\
\text { Mono: mean } \\
\text { aerosol model. }\end{array}$ & N/A & \\
\hline $\begin{array}{c}\text { Auger } \\
(8 \%) \\
{[17,21]}\end{array}$ & $\begin{array}{c}\text { Monthly models from } \\
\text { own radiosonde data. } \\
\text { Replaced by GDAS } \\
\text { with } 3 \mathrm{hr} \text { update. }\end{array}$ & $\begin{array}{l}4 \text { sites, hourly. Cloud } \\
\text { cover \& height, cross } \\
\text { check of VAOD. }\end{array}$ & $\begin{array}{l}2 \text { sites. Hourly } \\
\text { VAOD. Used } \\
\text { in analysis. }\end{array}$ & $\begin{array}{c}4 \text { sites, } 5 \text { min } \\
\text { scans. Data } \\
\text { selection via } \\
\text { LIDAR. }\end{array}$ & $\begin{array}{c}\text { aerosol } \\
\text { phase funct. } \\
\& \lambda \text { depend. } \\
\text { monitors. }\end{array}$ \\
\hline $\begin{array}{c}\mathrm{TA} \\
(11 \%) \\
{[22]}\end{array}$ & $\begin{array}{l}\text { Public data. Salt } \\
\text { Lake City \& Elko, } \\
200 \mathrm{~km} \text { from site }\end{array}$ & $\begin{array}{l}\text { One site (BR) twice } \\
\text { a day. Use fixed } \\
\text { model in recon } \\
\text { (average VAOD, } \\
\text { scale height, HAL) }\end{array}$ & $\begin{array}{l}\text { Hourly, will } \\
\text { use in analysis } \\
\text { in the future }\end{array}$ & $\begin{array}{l}1 \text { site, hourly } \\
\text { monitoring. } \\
\text { Used for data } \\
\text { selection. }\end{array}$ & \\
\hline
\end{tabular}

Each of the fluorescence observatories has a major program of atmospheric monitoring, outlined in Table 4. The molecular profile of the atmosphere may be determined by radiosonde launches or by data assimilation products like GDAS [21]. Aerosol conditions may be characterised by traditional LIDAR systems or by a bistatic LIDAR system employing a Central Laser Facility (CLF). Currently, the Auger Observatory uses molecular profiles from GDAS with 3-hour latency, hourly measurements of aerosols with its CLF and XLF, and sub-hourly measurements of cloud cover with LIDARs and infra-red cameras $[17,18]$. The Telescope Array uses publicly acquired radiosonde data, a fixed aerosol model derived from LIDAR observations, and infra-red cameras for cloud cover [22]. Hourly aerosol measurements will be used in the future as the CLF analysis matures.

\subsubsection{Photometric calibration}

Auger and the Telescope Array use different techniques for photometric calibration of their telescopes, and both nominate a significant $\sim 10 \%$ systematic uncertainty in energy due to calibration. Details of the calibration methods are given in [23] for Auger and [24] for TA. The TA experiment also has an opportunity for an end-to-end calibration with a electron linear accelerator system [25]. The WG sees an opportunity to inter-calibrate the two experiments in some way, perhaps using a flying calibrated light source (see below).

\subsubsection{Invisible energy corrections}

As discussed in Section 3.2, a correction must be applied to the calorimetric energy $E_{\text {cal }}$ measured by a fluorescence detector to account for invisible energy and to derive the primary energy $E_{0}$. The form of the correction is the same for Auger and TA, $E_{\mathrm{cal}} / E_{0}=A-B\left(E_{\mathrm{cal}} / \mathrm{EeV}\right)^{-C}$, but the constants $A, B, C$ are derived from different sources. The Auger values come from [26] assuming a QGSJET01 hadronic model, a mixed composition of 50:50 protons and iron, and a fixed $\theta=45^{\circ}$ zenith angle, resulting in $A=0.967, B=0.078$ and $C=0.140$. The Telescope Array group has performed their own CORSIKA simulations for a pure proton composition with the QGSJET-II model, and uniform arrival directions $\left(\theta<60^{\circ}\right)$ to extract $A=0.963, B=0.049$ and $C=0.181$. These curves are plotted in Figure 9. The separation between the Auger and TA correction is never larger than $2 \%$, and is in the opposite direction to that implied by the energy spectrum differences discussed in Section 4. In any case, the difference is well within the systematic uncertainty assigned to this correction by both experiments (Table 2). 


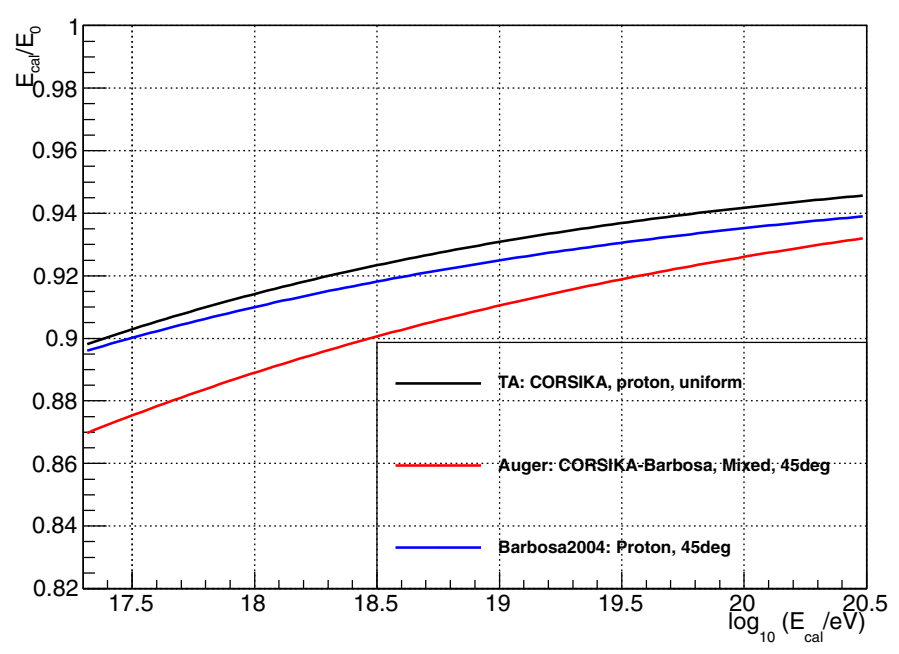

Figure 9. Comparison of the conversion factors from calorimetric energies to primary energies. See text for details.

\section{FUTURE DIRECTIONS}

In the future, each experiment will strive to reduce systematic uncertainties in energy calibration, attenuation curves, and reconstruction issues, and continue to employ cross-checks to validate these methods. Examples of cross-checks include using remote laser checks of photometric calibration, and hybrid data checks of surface signal attenuation with zenith angle. We will all continue to improve the important atmospheric monitoring and corrections.

As a community, we must maintain contacts between experiments. It may be necessary to have a high-level organization to encourage this, especially if more than a yearly meeting is desired. The WG supports the adoption of a world-wide fluorescence yield model, including humidity, pressure and temperature dependencies [16]. Where possible, we should use a common set of procedures. If some procedure is not the preferred one for a particular experiment, we suggest that it can be employed as a cross-check against the preferred method. For example TA could try to use the CIC method to crosscheck the MC-derived SD attenuation curve, and Auger could do the reverse. We should attempt to cross-check the TA and Auger FD photometric calibration, perhaps employing a calibrated light source flown by an octocopter, or by using a standard roving laser system. Finally, we can learn from each other about the best methods of atmospheric characterization.

\section{CONCLUSIONS}

The working group has examined the energy spectra and techniques of the currently operating experiments, the Pierre Auger Observatory, the Telescope Array, and Yakutsk, with some reference to previous measurements by AGASA and HiRes. We find that the spectra are in agreement after making energy scale shifts consistent with the quoted energy systematic uncertainties, with the possible exception of Yakutsk. After energy rescaling, the spectral normalizations and shapes are very consistent, and the positions of the spectral ankle and the suppression are in agreement.

The existence of the spectral ankle is certainly supported by all experiments. As is well known, the high-energy spectral suppression is not compatible with the AGASA spectrum, and the Yakutsk spectrum currently has insufficient exposure to properly detect it. But HiRes, TA and Auger all see the suppression with good statistical significance, with HiRes associating it with the GZK cut-off [27] because of their measurement of protonic composition at the highest energies, and the suppression's 
position in energy [8]. The Auger Observatory's measurement of mass composition at the highest energies is not consistent with protons under current hadronic interaction models [28], and so that collaboration has not yet associated the suppression with a particular mechanism.

We have started to investigate the alternative methods employed by the experiments. Essentially all analysis differences are based on well founded preferences, and should lead to equivalent outcomes. This should be tested in the future by cross-checks of methods using those alternatives. The energy scale difference seen between Auger and TA is consistent with systematic uncertainties, but does deserve further study. Common models (e.g. fluorescence yield) should be used where possible, and cross-checks of photometric calibrations and atmospheric corrections could be very instructive.

The members of the WG very much enjoyed their interactions which were played out in a friendly and constructive way. We hope that this process will be continued into the future to benefit all studies of ultra-high energy cosmic rays.

\section{References}

[1] F. Salamida et al. [Pierre Auger Collaboration], Proc. 32nd Int. Conf. Cosmic Rays 2 (2011) 145

[2] D. Ivanov, B.T. Stokes, G.B. Thomson et al. [Telescope Array Collaboration], Proc. 32nd Int. Conf. Cosmic Rays 2 (2011) 258., T. Abu-Zayyad et al.. arXiv:1205:5067 (submitted to Phys. Rev. Lett.)

[3] D. Ikeda et al. [Telescope Array Collaboration], Proc. 32nd Int. Conf. Cosmic Rays 2 (2011) 238

[4] Egorova V.P.,Glushkov A.V.,Knurenko S.P., et al., Nuclear Physics B (Proc.Suppl.) 136C (2004) 3; Ivanov A.A., Knurenko S.P., Pravdin M.I. and Sleptsov I.E., Moscow University Physics Bulletin, 2010 65, No 4 (2010) 292-299

[5] K. Shinozaki \& M. Teshima, Nuclear Physics B (Proc. Suppl.) 136 (2004) 18

[6] http://www.physics.rutgers.edu/ dbergman/HiRes-Monocular-Spectra-200702.html

[7] M. Unger, "EAS Studies of Cosmic Rays above $10^{16} \mathrm{eV}$ ", Proc. 32nd Int. Conf. Cosmic Rays 12 (2011)

[8] R.U. Abbasi et al. [Hires Collaboration], Phys. Rev. Lett. 100 (2008) 101101

[9] M. Takeda et al., Phys. Rev. Lett. 81 (1998) 1163

[10] L.G. Dedenko, D.A. Podgrudkov and T.M. Roganova, Physics of Atomic Nuclei 70 No. 10 (2007) 1759-1763

[11] R. Pesce et al. [Pierre Auger Collaboration], Proc. 32nd Int. Conf. Cosmic Rays 2 (2011) 214

[12] Y. Tsunesada et al., [Telescope Array Collaboration], Proc. 32nd Int. Conf. Cosmic Rays 2 (2011) 1270

[13] J. Allen et al. [Pierre Auger Collaboration], Proc. 32nd Int. Conf. Cosmic Rays 2 (2011) 83

[14] R. Abbasi et al., Astropart. Phys. 29 (2008) 77-86

[15] F. Kakimoto et al. Nucl. Instr. Meth. A 372 (1996) 527-533

[16] B. Keilhauer et al., "Nitrogen fluorescence in air for observing extensive air showers", this conference (2012)

[17] J. Abraham et al. [Pierre Auger Collaboration], Astropart. Phys. 33 (2010) 108-129

[18] B. Fick et al., JINST 1 (2006) 11003

[19] J.R. Vazquez et al., "The impact of the fluorescence yield on the energy reconstruction of UHECR showers", this conference (2012)

[20] R. Abbasi et al., Astropart. Phys. 25 (2006) 74

[21] P. Abreu et al. [Pierre Auger Collaboration], Astropart. Phys. 35 (2012) 591-607

[22] T. Tomida et al., Nucl. Instr. Meth. A 654 (2011) 653-660, Y. Takahashi et al., UHECR2010, AIP Conf. Proc. 1367, 157 (2011), F. Shibata et al., UHECR2010, AIP Conf. Proc. 1367, 165 (2011), Y. Kobayashi et al., UHECR2010, AIP Conf. Proc. 1367, 169 (2011)

[23] J. Abraham et al. [Pierre Auger Collaboration], Nucl. Instr. Meth. A 620 (2010) 227-251 


\section{UHECR 2012}

[24] S. Kawana et al., Nucl. Instr. Meth. A 681 (2012) 68-77, H. Tokuno et al. Nucl. Instr. Meth. A 601 (2009) 364-371

[25] T. Shibata et al., Proc. 31st Int. Conf. Cosmic Rays (2009); T. Shibata et al., Nucl. Instr. Meth. A 597 (2008) 61., T. Shibata et al., UHECR2010, AIP Conf. Proc. 1367, 44 (2011)

[26] H. Barbosa et al., Astropart. Phys. 22 (2004) 159

[27] K. Greisen, Phys. Rev. Lett. 16 (1966) 748; G.T. Zatsepin and V.A. Kuz'min, Pis'ma Zh. Eksp. Teor. Fiz 4 (1966) 114 [JETP Lett. 4 (1966) 78]

[28] E. Barcikowski et al., "Mass Composition Working Group Report", this conference (2012) 